\title{
Serious Games e Neurofeedback como alternativa de tratamento de indivíduos com Transtorno de Déficit de Atenção e Hiperatividade: uma revisão sistemática
}

\author{
Giulia T. Monteiro e Diana F. Adamatti \\ Centro de Ciências Computacionais - Universidade Federal do Rio Grande (FURG) \\ Caixa Postal 474 - 96.203-900 - Rio Grande - RS - Brazil \\ \{giuliatondin, dianaadamatti\}@furg.br

\begin{abstract}
This paper presents a systematic review of the use of Serious Games and Neurofeedback as a support mechanism for the treatment of individuals with Attention Deficit Hyperactivity Disorder (ADHD). The review aims to present the methodologically strongest results in this area and to verify whether this alternative is accessible, applicable and effective. This way, the proposed solutions are described and analyzed, pointing out the significant characteristics and limitations of these studies. Results demonstrate the great potential that this technology has as an instrument for monitoring and engagement, as well as providing a reliable and accessible method for cognitive improvement, being a great approach for the social and academic development of individuals with ADHD.
\end{abstract}

Resumo. Este artigo apresenta uma revisão sistemática sobre a utilização de Serious Games e Neurofeedback como mecanismo de apoio para o tratamento de indivíduos com Transtorno de Déficit de Atenção e Hiperatividade (TDAH). A revisão busca apresentar os resultados metodologicamente mais fortes dessa área e verificar se essa alternativa é acessível, aplicável e eficaz. Desse modo, são descritas e analisadas as soluções propostas, apontando as características significativas e as limitações desses estudos. Os resultados demonstraram o grande potencial que essa tecnologia possui como um instrumento de acompanhamento e engajamento, além de fornecer um método confiável e acessível para o aperfeiçoamento cognitivo, sendo uma grande aliada para o desenvolvimento social e acadêmico de indivíduos com TDAH.

\section{Introdução}

O Transtorno de Déficit de Atenção e Hiperatividade (TDAH) é considerado um transtorno do neurodesenvolvimento que engloba em sua sintomatologia déficits relacionados a interação e habilidades de comunicação, manifestados desde a infância [Jou et al. 2010]. Esse transtorno apresenta um padrão de desatenção e/ou hiperatividade-impulsividade que afeta o funcionamento e o desenvolvimento, tanto social, acadêmico ou profissional do indivíduo.

Os sintomas de TDAH se manifestam em 95\% dos casos antes dos 12 anos [Neurosaber 2016], trazendo grandes prejuízos para o aprendizado logo na fase escolar. De acordo com a neuropsicologia, um dos maiores déficits que essa população possui e que afetam o seu desenvolvimento apresenta-se nas funções cognitivas das diferentes categorias de atenção, sendo elas: atenção seletiva, concentrada, sustentada e alternada 
[Coutinho et al. 2007]. Os déficits de atenção que esses indivíduos possuem em diferentes graus podem comprometer a vida escolar dos mesmos e torná-los menos capacitados para o mercado de trabalho [Souza et al. 2007, Castro and Lima 2018].

O TDAH não possui cura até o momento, entretanto os tratamentos realizados buscam diminuir a intensidade e a frequência dos sintomas, permitindo uma melhor qualidade de vida, possuindo a tecnologia como uma aliada. Uma das soluções que vem auxiliando a neuropsicologia nesse âmbito são os Serious Games (SG). Esses são capazes de estimular as funções cognitivas, melhorando o desempenho dos indivíduos que os praticam, ao mesmo tempo que integram princípios de aprendizagem [Savi and Ulbricht 2008]. Evidências apontam que a reabilitação neuropsicológica pode ser mais eficaz do que tratamentos realizados a partir de medicações [Oliveira et al. 2013], evitando os efeitos colaterais do uso excessivo de remédios.

Para um melhor controle e eficácia sobre o aprimoramento das funções cognitivas fornecido pelos Serious Games, pode ser utilizada uma Brain-Computer Interface (BCI), um sistema baseado em Neurofeedback. Essa tecnologia possui como objetivo principal controlar algum dispositivo, através de sinais elétricos detectados no escalpo, na superfície cortical ou áreas subcorticais cerebrais, a fim de estabelecer uma via de comunicação com o sistema nervoso central (SNC) que seja independente das vias padrão [Barbosa 2012].

Para efetivar o controle de algum dispositivo através da BCI podem ser utilizados dois métodos: invasivo e não invasivo. O primeiro baseia-se em neuroimplantes, ou seja, eletrodos implantados dentro da caixa craniana, fornecendo sinais de melhor qualidade do que o segundo método, porém maior risco [Barbosa 2012]. O segundo normalmente é realizado através de um eletroencefalograma (EEG) que provê uma comunicação simples para registro dos sinais, captando a atividade elétrica cerebral espontânea através de eletrodos aplicados sobre o couro cabeludo.

O baixo custo e a portabilidade de dispositivos EEG permitiram a utilização do mesmo juntamente com SG para apoiar diversas soluções que buscam lidar com déficits cognitivos de atenção. $\mathrm{O}$ atual estudo tem como objetivo realizar uma revisão sistemática para investigar e sintetizar soluções produzidas que utilizam essas tecnologias para um tratamento alternativo de indíviduos com TDAH, de modo a delinear o que está sendo realizado nesta área de pesquisa e verificar se essas ferramentas são acessíveis, aplicáveis e eficazes. Para isso, será conduzido um protocolo para Revisão Sistemática da Literatura (RSL), de modo a destacar os resultados metodologicamente mais fortes e fornecer informações sobre a aplicabilidade dos efeitos sustentáveis dessas soluções.

\subsection{Potencial da tecnologia}

Os benefícios da utilização de SG aliados com BCI como alternativa para o tratamento de TDAH vão além da substituição do tratamento tradicional de medicação, mas também proporcionam uma ferramenta confiável, com feedbacks constantes sobre o avanço do indivíduo, e agradável, envolvendo com facilidade o paciente no tratamento e nos exercícios cognitivos.

Visto que a maioria dos casos de TDAH são identificados ainda na infância, a utilização dessa solução demonstra-se um modo mais efetivo para gerar engajamento em crianças do que outras terapias [Lee et al. 2015]. Aplicar SGs juntamente com BCI per- 
mite utilizar os diferentes tipos de atenção necessárias no cotidiano e na sala de aula como mecanismo de jogabilidade. Esse engajamento leva ao aprimoramento da atenção e pode ser utilizado como estratégia no processo de ensino-aprendizagem de estudantes com TDAH.

Porém para conseguir atingir todo o potencial desejado fornecido por essa tecnologia e beneficiar os pacientes e usuários da melhor maneira possível, é necessário que essa linha de pesquisa evolua a partir de estudos baseados em evidências devidamente validadas, valorizando uma base teórica forte, condução de estudos randomizados controlados, além de analisar os resultados pré e pós intervenção ao longo do tempo [Lee et al. 2015].

Por essa razão, para aumentar a confiabilidade dos resultados sintetizados nessa revisão, foram descartados estudos que não possuiam em sua metodologia voluntários diagnosticados com TDAH, porém vale destacar as soluções propostas por [Thomas et al. 2013, Rohani and Puthusserypady 2015, Monaco et al. 2018, Barba et al. 2019], que apesar de serem estudos pilotos e não realizar os testes com essa população, descrevem alternativas interessantes dentro desse tema de pesquisa.

\section{Protocolo da Revisão Sistemática}

Visto o objetivo principal da revisão, podemos estabelecer a questão que guiará os passos da pesquisa: quais estudos foram desenvolvidos para avaliar a eficácia da aplicação de Serious Games (SG) aliados a Brain-Computer Interface (BCI) como suporte para o tratamento de indivíduos com Transtorno de Déficit de Atenção e Hiperatividade (TDAH)?

\subsection{Estratégia de pesquisa}

A busca pelos estudos foi realizada nas maiores bases de dados das áreas de computação e ciências da saúde, sendo elas: Science Direct ${ }^{1}$, SpringerLink $^{2}$, IEEEXplore ${ }^{3}$, Scopus $^{4}$, SciELO $^{5}$ e PubMed Central (PMC) ${ }^{6}$. Na base brasileira SciELO, nenhum resultado foi obtido, ou seja, nenhum trabalho em português foi encontrado, a partir disso todos os termos de busca foram definidos em inglês.

As palavras-chaves definidas foram: ("attention deficit" OR "hyperactivity disorder" OR "ADHD”) AND ("Serious games") AND ("Brain-computer interface” OR "BCI" OR "EEG-based" OR "Brain-machince interface” OR “BMI”). Além disso, foram selecionadas apenas as pesquisas realizadas a partir de 1 de janeiro de 2010 até o momento atual, de modo a sintetizar os resultados mais atuais e avançados da área.

\subsection{Processo de seleção}

Com o intuito de refinar os resultados obtidos a partir da decomposição em artigos relevantes e irrelavantes para a pesquisa, foram definidos critérios de inclusão e exclusão para serem aplicados na seleção dos estudos, conforme descrito na Tabela 1.

\footnotetext{
${ }^{1}$ https://www.sciencedirect.com/

${ }^{2}$ https://link.springer.com/

${ }^{3}$ https://ieeexplore.ieee.org/Xplore/home.jsp

${ }^{4}$ https://www.scopus.com/

${ }^{5}$ https://www.scielo.br/

${ }^{6}$ https://www.ncbi.nlm.nih.gov/pmc/
} 
IX Congresso Brasileiro de Informática na Educação (CBIE 2020)

Anais do XXXI Simpósio Brasileiro de Informática na Educação (SBIE 2020)

Tabela 1. Critérios de Inclusão e Exclusão

\begin{tabular}{|c|c|}
\hline Critérios de Inclusão (CI) & Critérios de Exclusão (CE) \\
\hline CI 1: Iniciativas de pesquisas relacionadas & $\begin{array}{l}\text { CE 1: Trabalhos que não utilizam SG aliados } \\
\text { com BCI. }\end{array}$ \\
\hline $\begin{array}{c}\text { ferramenta de suporte para tratamento de } \\
\text { indivíduos com TDAH. }\end{array}$ & $\begin{array}{c}\text { CE 2: Pesquisas que não utilizam população } \\
\text { diagnosticada. }\end{array}$ \\
\hline CI 2: Estudos publicados após 2010. & $\begin{array}{c}\text { CE 3: Trabalhos que não trazem resultados } \\
\text { a partir de testes. }\end{array}$ \\
\hline
\end{tabular}

O diagrama de fluxo da pesquisa e seleção pode ser observado na Figura 1. A busca dos termos nas bases de dados retornaram 319 estudos, baseando-se nos critérios de inclusão definidos. A partir disso, primeiramente, todos os critérios de exclusão foram utilizados para analisar as informações obtidas de cada estudo a partir da leitura do título e resumo dos mesmos, resultando em uma exclusão de 291 pesquisas, passando apenas 28 para a próxima etapa de refinamento. Após a leitura da Introdução dos estudos, foi aplicado o critério de exclusão 1, passando apenas 12 estudos para a segunda etapa. Por fim, foram aplicados os critérios de exclusão 2 e 3 nas pesquisas a partir da análise do texto completo, com ênfase na metodologia e resultados, finalizando a seleção em apenas 6 estudos.

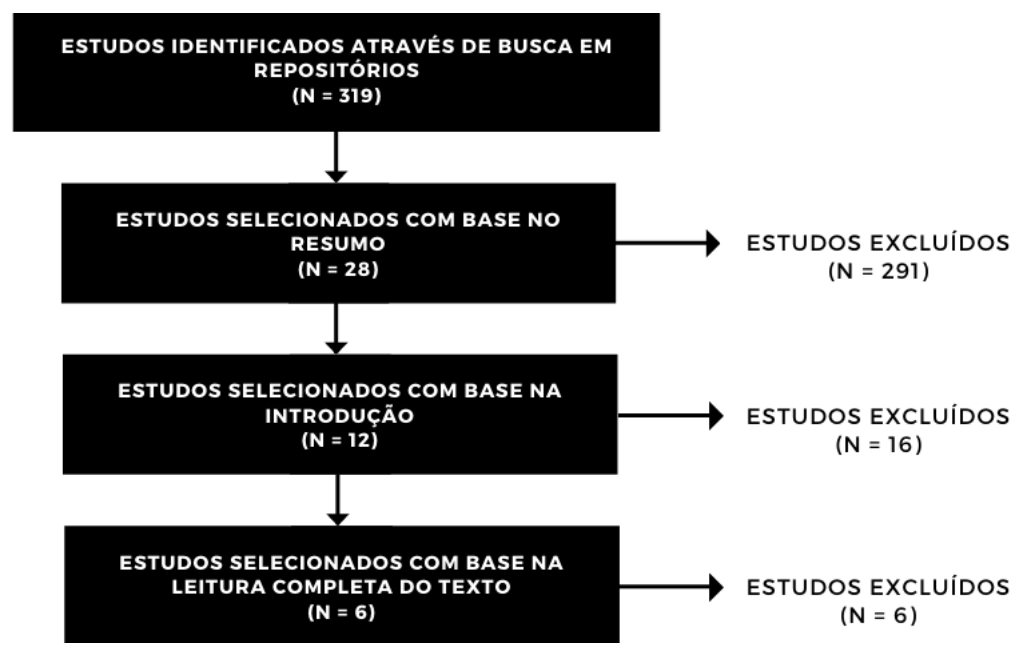

Figura 1. Fluxograma do processo de seleção dos estudos

A grande quantidade de estudos retornados inicialmente pelas bases de dados é justificada pela inclusão de artigos que não fazem parte da questão fundamental da pesquisa. Esses artigos foram selecionados pelo repositório SpringerLink, que não levou em consideração em muitos estudos a necessidade de ser uma solução de SG para uma população com TDAH, porém foram devidamente identificados a partir dos critérios definidos e removidos.

\section{Estudos selecionados}

O estudo de [Lim et al. 2012] propõe um controle baseado no nível de atenção de crianças com TDAH para determinar a velocidade de um avatar em um jogo, a partir da captação 
dos sinais cerebrais. O estudo aprimorou a função cognitiva de atenção de 20 participantes ao longo de 8 semanas de treinamento, além de melhorar os comportamentos de hiperatividade-impulsividade dos indivíduos analisados. O acompanhamento póstratamento foi definido como uma "fase de manutenção", onde ocorreram treinamentos de reforço por 3 meses consecutivos. A solução foi testada novamente com pequenas alterações na metodologia no estudo de [Lee et al. 2015]. Um total de 160 voluntários foram dividos em dois grupos e a avaliação foi realizada a partir de um método cego por médicos, completando os resultados anteriormente obtidos.

Já [Blandon et al. 2016] desenvolveram um jogo em Realidade Virtual (RV) imersiva para o treinamento da autorregulação do nível de atenção ao focar em determinados itens durante o jogo. Os sinais cerebrais emitidos ao indivíduo autoregulam diferentes graus de atenção que são captados pela BCI e o jogo só avança se esses sinais estiverem de acordo com o nível necessário pré-determinado. Ao todo, duas sessões de intervenção foram aplicadas em 9 crianças com TDAH e os resultados obtidos foram capazes de demonstrar a melhora na performance do jogo ao longo do tempo, que refletiu no aprimoramento de habilidades de manutenção da atenção sustentada.

Enquanto [Alchalabi et al. 2018] investigaram a eficácia de um SG aliado com BCI capaz de detectar TDAH a partir da utilização paralela de um classificador de $M a$ chine Learning que analisa os sinais de atenção durante o jogo. A proposta foi testada com 9 jovens-adultos, onde 4 possuíam TDAH e 5 eram neurotípicos (aqueles não possuem condições neurológicas específicas), durante 10 a 12 sessões, variando em relação ao grupo analisado. A solução obteve $98 \%$ de acurácia na detecção do estado de atenção, obtendo resultados satisfatórios.

O estudo de [Mercado et al. 2018] analisou a usabilidade, a experiência de usuário e o nível de atenção sustentada ao utilizar um jogo para treinamento com neurofeedback com 12 crianças com TEA (Transtorno do Espectro Autista) e TDAH comórbida. O estudo aplicou 2 sessões para cada voluntário, onde foi possível observar questões de acessibilidade, além de aumentar o tempo de atenção sustentada dos voluntários durante o jogo.

Por fim, [Park et al. 2018] buscaram melhorar a habilidade de leitura de crianças com TDAH a partir do desenvolvimento de um jogo baseado em uma narrativa de conto de fadas e atividades de atenção e de comportamento propostas durante o mesmo. Para avançar no jogo ao realizar atividades de atenção, o jogador precisa manter o foco de modo que a BCI capte os sinais necessários de atenção, realizando o treinamento da atenção sustentada. Os testes foram realizados com 5 crianças durante 5 sessões. Os resultados demonstraram melhoras na leitura em voz alta e na compreensão da mesma, além de aumentar o tempo de atenção sustentada e diminuição do comportamento hiperativo ao longo das sessões.

Na Tabela 2 é apresentada uma visão geral dos artigos selecionados, com o objetivo de sintetizar as informações básicas de cada um.

\section{Análise dos estudos}

Esta seção apresenta alguns tópicos relevantes para a análise e comparação dos estudos selecionados, aprofundando questões relacionadas ao objetivo e tipo de cada solução, 
IX Congresso Brasileiro de Informática na Educação (CBIE 2020)

Anais do XXXI Simpósio Brasileiro de Informática na Educação (SBIE 2020)

Tabela 2. Visão geral dos estudos selecionados

\begin{tabular}{|c|c|c|c|c|c|}
\hline Referência & País & Objetivo & Voluntários & Sessões & Resultados \\
\hline $\begin{array}{l}\text { [Lim et al. 2012] } \\
\text { [Lee at al. 2015] }\end{array}$ & Singapura & $\begin{array}{l}\text { Aprimorar função } \\
\text { cognitiva de atenção }\end{array}$ & $\begin{array}{l}20 \text { crianças no primeiro, } \\
\text { e } 160 \text { crianças no } \\
\text { segundo }\end{array}$ & 24 sessões & $\begin{array}{l}\text { Melhora do nível de atenção } \\
\text { e de comportamentos de } \\
\text { hiperatividade-impulsividade }\end{array}$ \\
\hline [Blandon et al. 2016] & Colômbia & $\begin{array}{c}\text { Aperfeiçoar a } \\
\text { a capacidade de } \\
\text { autoregulação da } \\
\text { atenção } \\
\end{array}$ & 9 crianças & 2 sessões & $\begin{array}{l}\text { Aprimoramento de habilidades } \\
\text { de manutenção da atenção } \\
\text { sustentada }\end{array}$ \\
\hline [Alchalabi et al. 2018] & Canadá & $\begin{array}{c}\text { Facilitar a detecção } \\
\text { de TDAH }\end{array}$ & $\begin{array}{l}4 \text { jovens-adultos } \\
\text { com TDAH } \\
\text { e } 5 \text { neurotípicos }\end{array}$ & $\begin{array}{l}12 \text { sessões para } \\
\text { indivíduos com } \\
\text { TDAH e } 10 \\
\text { para neurotípcos. }\end{array}$ & $\begin{array}{c}\text { Capaz de detectar } \\
\text { o estado de atenção e } \\
\text { classificar o TDAH com } \\
98 \% \text { de acurácia }\end{array}$ \\
\hline [Mercado et al. 2018] & México & $\begin{array}{c}\text { Melhorar a atenção } \\
\text { sustentada e analisar } \\
\text { a usabilidade e } \\
\text { experiência de } \\
\text { usuário }\end{array}$ & $\begin{array}{l}12 \text { crianças com } \\
\text { TDAH e TEA }\end{array}$ & 2 sessões & $\begin{array}{l}\text { Aumento do nível de ateção } \\
\text { sustentada, além de ser } \\
\text { avaliado como fácil de usar, } \\
\text { útil e divertido }\end{array}$ \\
\hline [Park et al. 2018] & Coréia do Sul & $\begin{array}{l}\text { Melhorar a habili- } \\
\text { dade de leitura e de } \\
\text { atenção }\end{array}$ & 5 crianças & 5 sessões & $\begin{array}{l}\text { Melhora no tempo de atenção } \\
\text { sustentada, na leitura em voz } \\
\text { alta e na compreensão do texto, } \\
\text { além da diminuição de } \\
\text { comportamento hiperativo. }\end{array}$ \\
\hline
\end{tabular}

além da verificação dos efeitos entre grupos de controle. Por fim, serão apontadas as limitações encontradas de cada estudo e as direções futuras.

\subsection{Objetivo da solução}

Podemos dizer que cada estudo teve um objetivo geral diferente, porém foi possível identificar uma forte correlação entre parte dos objetivos específicos de 5 dos 6 estudos, que investigaram o aprimoramento da atenção sustentada. O grande foco nesse tópico é justificado pelo efeito direto que o mesmo possui nos processos relacionados à aprendizagem, visto que ele define a capacidade de focar em uma atividade contínua e repetitiva em um longo período e sem distrações [Coutinho et al. 2007]. Diferentes níveis dessa atenção demandam tempo e esforços mentais diferentes, além disso, podem afetar a autoconfiança do indivíduo.

Em [Lim et al. 2012, Lee et al. 2015, Mercado et al. 2018] o aprimoramento de habilidades de atenção sustentada é o objetivo principal, porém [Mercado et al. 2018] também busca verificar como o esforço demandado dessa atenção afeta a experiência do usuário, em termos de facilidade e atratividade da solução, e também sua usabilidade.

Já em [Blandon et al. 2016], o objetivo vai além do aprimoramento da atenção sustentada, ele busca aperfeiçoar a capacidade de autorregulação da atenção pelo indivíduo, ou seja, a habilidade de regular e até mesmo induzir um estado de atenção específico, levando em conta o intervalo de tempo entre o estímulo e a resposta.

Enquanto [Park et al. 2018] busca aprimorar a habilidade de leitura e diminuir comportamentos hiperativos, baseando-se também na aplicação da atenção sustentada para realizar as atividades propostas. Esse objetivo é definido pelos autores visto as dificuldades enfrentadas por essa população no processo de aprendizagem da leitura, que podem causar baixo rendimento acadêmico e problemas sociais no âmbito escolar.

Por fim, diferenciando-se das propostas anteriores, [Alchalabi et al. 2018] detectam os estados de atenção com o objetivo de facilitar o diagnóstico de pessoas com TDAH. A proposta busca ajudar no processo de detecção, entretendo o paciente e diminuindo o efeito do "jaleco branco", uma reação que o indivíduo tem ao estar em frente ao médico, 
IX Congresso Brasileiro de Informática na Educação (CBIE 2020)

Anais do XXXI Simpósio Brasileiro de Informática na Educação (SBIE 2020)

principalmente em crianças que podem enxergar o profissional como um punidor em testes realizados tradicionalmente.

Todas as soluções propostas definiram seus objetivos baseando-se na utilização de tecnologias acessíveis de EEG, para que as mesmas pudessem ser consideradas alternativas aplicáveis e de baixo-custo para o tratamento de indivíduos com TDAH.

\subsection{Tipo de controle do Serious Game}

De modo geral, os estudos basearam-se em dois tipos de controle: controle por nível de atenção e controle por estado mental e de atenção específico. O primeiro tipo foi utilizado nos estudos de [Lim et al. 2012, Lee et al. 2015, Blandon et al. 2016, Mercado et al. 2018, Park et al. 2018], enquanto o segundo, no estudo de [Alchalabi et al. 2018].

O primeiro tipo caracteriza-se pela utilização de diferentes níveis de atenção sustentada e seletiva (habilidade em focar em determinado lugar, apesar de fatores distrativos) para atingir um determinado objetivo, como mover o avatar do jogo nos estudos de [Lim et al. 2012, Lee et al. 2015, Mercado et al. 2018] ou mirar e selecionar objetos da simulação nos estudos de [Blandon et al. 2016, Park et al. 2018].

Na movimentação, o nível de atenção determina a velocidade do avatar, onde quanto mais focado o jogador está, mais rápido ele movimenta-se, assim como quanto mais desatento, mais lentamente ele movimenta-se. No estudo de [Mercado et al. 2018], caso a velocidade do avatar diminuia consideravelmente, também são utilizados sinalizações sonoras e visuais para que o jogador volte sua atenção para o jogo.

Na seleção a partir do nível de atenção, uma atividade do jogo só é considerada completa se o jogador conseguir manter um determinado nível de atenção durante uma parcela de tempo pré-definida. Por exemplo, no estudo de [Park et al. 2018], onde uma atividade solicita que o usuário foque na personagem do livro para que a mesma consiga realizar a ação. Enquanto que no estudo de [Blandon et al. 2016] é solicitado que o usuário aumente seu nível de atenção de modo incremental (alcançando 60\%, 65\%, 70\%, $75 \%, 80 \%$ e $100 \%$ da mesma) para realizar a ação.

O segundo tipo é utilizado a partir da captação de sinais cerebrais específicos de determinados estados mentais e de atenção para realizar as ações do jogo. Em [Alchalabi et al. 2018] é solicitado que o jogador concentre-se e treine algumas imagens mentais pré-determinadas, como a imagem do avatar empurrando uma caixa. Os sinais cerebrais emitidos ao imaginar essa imagem são gravados e o jogador só conseguirá realizar essa mesma ação no jogo, caso o seu nível de atenção seja suficiente para que o estado metal de controle seja reconhecido.

O primeiro tipo provou sua eficácia no aprimoramento em atividades de estimulação cognitiva, realizando o aperfeiçoamento da atenção nessa população. Enquanto o segundo demonstrou ser um método confiável e eficaz para a realização rápida de diagnósticos de TDAH.

\subsection{Efeitos entre grupos de controle}

Podemos dizer que 5 dos 6 estudos possuem efeitos similares em seus grupos de controle, ou seja, impactos semelhantes não específicos de uma determinada solução, mas 
do tipo de controle utilizado no desenvolvimento do SG aliado ao BCI. [Lim et al. 2012, Lee et al. 2015, Blandon et al. 2016, Mercado et al. 2018, Park et al. 2018] aprimoraram o nível de atenção de seus participantes de forma efetiva e consideravelmente rápida.

Ainda que os efeitos sejam similares no aprimoramento cognitivo de atenção, podemos dizer que algumas soluções são mais efetivas do que outras, apesar de utilizar o mesmo tipo de controle. No estudo de [Mercado et al. 2018], os níveis de atenção obtidos são comparados em dois SGs diferentes que utilizam a mesma configuração no BCI. Os resultados diferentes no nível de atenção são justificados pela experiência do usuário utilizando a solução, a sua usablilidade e o grau de empatia que o jogador possui pelo personagem do jogo, que são fatores importantes ao desenvolver uma proposta de SG.

Além disso, nos estudos de [Lim et al. 2012, Lee et al. 2015, Park et al. 2018], a proposta e o tipo de controle também resultou na diminuição de comportamentos hiperativos, ao aperfeiçoar as habilidades de atenção sustentada e seletiva.

\subsection{Limitações encontradas e direções futuras}

No estudo de [Lim et al. 2012] as principais limitações encontradas foram em relação a aplicação do estudo. Ele foi realizado como um open-label trial, ou seja, quando as informações da intervenção não são retidas dos participantes dos estudos, o que pode causar viés nos resultados. Além disso, as avaliações de comportamento foram realizadas pelos pais e as informações dadas pelos professores não foram suficientes para serem consideradas. Por isso, foi apontada a importância de realizar um estudo randomizado controlado para avaliar mais efetivamente a eficácia da solução.

Deste modo, no estudo de [Lee et al. 2015], que busca aprimorar a metodologia da intervenção proposta por [Lim et al. 2012], a solução foi aplicada em dois grupos para a realização de testes randomizados controlados. Porém, o conteúdo do jogo foi considerado um limitador em questão, visto que não existe muita variação, mesmo possuindo 3 níveis diferentes de dificuldade, e por ser algo repetitivo, pode ter afetado os resultados, pois algumas crianças evoluíram relativamente rápido no jogo. Em vista disto, como direções futuras os autores afirmaram a necessidade de um maior estudo da complexidade do software da intervenção BCI e do perfil dos indivíduos que são beneficiados.

[Blandon et al. 2016] não apontaram limitações, mas colocaram luz sobre a necessidade de uma melhor condução do estudo, utilizando grupos de controle para comparar os resultados, uma maior análise sistemática, além de uma intervenção mais prolongada, com no mínimo 10 sessões, para obter resultados com maior acurácia. Como trabalhos futuros, apontam o potencial da captação e utilização de diferentes potências de banda, para otimizar os efeitos de terapias baseadas em jogos com neurofeedback.

Do mesmo modo, [Alchalabi et al. 2018] também não apontaram limitações, sendo um estudo que desenvolveu uma forte base para a criação e viabilização de jogos para a detecção de TDAH. É descrito o potencial da utilização da Realidade Virtual para enriquecer a interação e gerar engajamento em soluções futuras.

A principal limitação descrita no estudo de [Mercado et al. 2018] refere-se ao tamanho da amostra utilizada para os testes, que por ser pequena dificulta a generalização dos resultados. Além disso, o pequeno número de sessões aplicadas em cada voluntário limitou a análise dos efeitos a longo prazo e do engajamento frente a solução. Como pro- 
posta futura, é colocado em questão a investigação de como a personagem do jogo pode afetar o comportamento e as emoções do jogador.

Por fim, [Park et al. 2018] também descreveu como limitação o pequeno número da amostra para generalização dos resultados dos testes. Como direção futura do estudo, foi descrito o potencial da utilização de tecnologias como biosensores e wearable devices para integração com esse tipo de solução.

\section{Conclusão}

A utilização de Serious Games aliados a tecnologias não invasivas de Brain-Computer Interface como alternativa de tratamento para reabilitação neuropsicológica na população com Transtorno de Déficit de Atenção e Hiperatividade possui grande potencial, melhorando as funções cognitivas dos indivíduos paralelamente a aplicação de princípios de aprendizagem e comportamento.

Esse meio de intervenção, além de possuir baixo custo e acessibilidade, faz com que o indíviduo se engaje com facilidade no tratamento e nos exercícios cognitivos, ao mesmo tempo que contorna os efeitos colaterais do uso excessivo de remédios e fornece um método confiável e eficaz, onde seus avanços podem ser acompanhados através de feedbacks constantes. Além disso, essa tecnologia demonstrou ser um método aplicável, efetivo e rápido para a realização de diagnósticos de TDAH.

Para que todo o potencial dessa tecnologia seja aproveitado, é necessário que as pesquisas para o avanço dessa área se baseiem em metodologias com uma forte base de conhecimento e procedimentos válidos para a análise dos benefícios efetivos e reais nessa população. São recomendadas aplicações de estudos randomizados controlados para uma comparação com maior acurácia dos resultados, além da utilização de amostras maiores de voluntários e acompanhamento pós intervenção para a generalização dos resultados. Esse trabalho buscou realizar uma revisão sistemática sobre os estudos desenvolvidos nessa área, para que fosse possível reunir as abordagens já realizadas e contribuir na consolidação de metodologias eficazes no desenvolvimento dessa essencial ferramenta de apoio.

\section{Referências}

Alchalabi, A. E., Shirmohammadi, S., Eddin, A., and Elsharnouby, M. (2018). Focus: Detecting adhd patients by an eeg-based serious game. IEEE Transactions on Instrumentation and Measurement, PP:1-9.

Barba, M., Covino, A., De Luca, V., De Paolis, L., D'Errico, G., Bitonto, P., Gestore, S., Magliaro, S., Nunnari, F., Paladini, G., Potenza, A., and Schena, A. (2019). Bravo: A gaming environment for the treatment of adhd. In Augmented Reality, Virtual Reality, and Computer Graphics, pages 394-407.

Barbosa, A. F. (2012). Um sistema inteligente de classificação de sinais de eeg para interface cérebro-computador. Master's thesis, Programa de Pós-Graduação em Engenharia Elétrica e de Computação - Universidade Federal do Rio Grande do Norte.

Blandon, D., Muñoz, J., Lopez, D., and Henao, O. (2016). Influence of a bci neurofeedback videogame in children with adhd. quantifying the brain activity through an eeg signal processing dedicated toolbox. In 2016 IEEE 11th Colombian Computing Conference (CCC), pages 1-8. 
IX Congresso Brasileiro de Informática na Educação (CBIE 2020)

Anais do XXXI Simpósio Brasileiro de Informática na Educação (SBIE 2020)

Castro, C. and Lima, R. (2018). Consequências do transtorno do déficit de atenção e hiperatividade (tdah) na idade adulta. Revista Psicopedagogia, 35:61-72.

Coutinho, G., Mattos, P., Araújo, C., and Duchesne, M. (2007). Transtorno do déficit de atenção e hiperatividade: contribuição diagnóstica de avaliação computadorizada de atenção visual. Revista De Psiquiatria Clinica - REV PSIQUIATR CLIN, 34.

Jou, G., Amaral, B., Riota, C., Schaefer, L., and Zimmer, M. (2010). Transtorno de déficit de atenção e hiperatividade: um olhar no ensino fundamental. Psicologia Em Estudo, 23.

Lee, X., Koukouna, E., Lim, C., Guan, C., Lee, T., and Fung, D. (2015). Can we play with adhd? an alternative game-based treatment for inattentive symptoms in attention-deficit/hyperactivity disorder. In Subconscious Learning via Games and Social Media, pages 69-86, Singapore. Springer Singapore.

Lim, C., Lee, T., Guan, C., Fung, D., Zhao, Y., Teng, S., Zhang, H., and Krishnan, K. (2012). A brain-computer interface based attention training program for treating attention deficit hyperactivity disorder. PloS one, 7.

Mercado, J., Espinosa Curiel, I., Escobedo, L., and Tentori, M. (2018). Developing and evaluating a bci video game for neurofeedback training: the case of autism. Multimedia Tools and Applications, 78 .

Monaco, A., Sforza, G., Amoroso, N., Antonacci, M., Bellotti, R., de Tommaso, M., Bitonto, P., Di Sciascio, E., Diacono, D., Gentile, E., Montemurno, A., Ruta, M., Ulloa, A., and Tangaro, S. (2018). The person project: a serious brain-computer interface game for treatment in cognitive impairment. Health and Technology, 9:1-11.

Neurosaber (2016). Características de jovens e crianças com tdah. https://bit.ly/ 2t 81Xph. Acesso em: 07-07-2020.

Oliveira, L., Ishitani, L., and Cardoso, A. (2013). Jogos computacionais e transtorno de déficit de atenção e hiperatividade: Revisão sistemática de literatura. Taller Internacional de Software Educativo.

Park, K. E., Kihl, T., Park, S., Kim, M.-J., and Chang, J. (2018). Fairy tale directed game-based training system for children with adhd using bci and motion sensing technologies. Behaviour Information Technology, 38:1-14.

Rohani, D. and Puthusserypady, S. (2015). Bci inside a virtual reality classroom: a potential training tool for attention. EPJ Nonlinear Biomedical Physics, 3.

Savi, R. and Ulbricht, V. (2008). Jogos digitais educacionais: Beneficios e desafios. RENOTE, 6.

Souza, I., Serra-Pinheiro, M., Fortes, D., and Pinna, C. (2007). Dificuldades no diagnóstico de tdah em crianças. Jornal Brasileiro De Psiquiatria, 56.

Thomas, K. P., Vinod, A., and Guan, C. (2013). Design of an online eeg based neurofeedback game for enhancing attention and memory. Annual International Conference of the IEEE Engineering in Medicine and Biology Society, 2013:433-436. 